\title{
GAD65 autoantibodies in women with gestational or insulin dependent diabetes mellitus diagnosed during pregnancy
}

\author{
J.S. Petersen ${ }^{1}$, T. Dyrberg ${ }^{2}$, P. Damm ${ }^{3}$, C. Kühl' ${ }^{2}$ L. Mølsted-Pedersen ${ }^{3}$, K. Buschard ${ }^{4}$ \\ ${ }^{1}$ Hagedorn Research Institute, Gentofte, Denmark \\ ${ }^{2}$ Novo Nordisk A/S, Bagsværd, Denmark \\ ${ }^{3}$ Bartholin Instituttet, Kommunehospitalet, Copenhagen, Denmark \\ ${ }^{4}$ Diabetes Centre, Department of Obstetrics and Gynaecology, University of Copenhagen, Denmark
}

\begin{abstract}
Summary We have studied the presence of GAD65 autoantibodies in women with insulin-dependent diabetes mellitus (IDDM) $(n=28)$ or gestational diabetes $(\mathrm{GDM})(n=139)$ diagnosed during pregnancy and investigated the temporal relationship between these autoantibodies and the subsequent recurrence or development of IDDM. Among the GDM patients, $4.3 \%$ (6 of 139) developed true IDDM during a median follow-up period of 6.3 years (range 4.0 11.0). Of these, $2.2 \%$ (3 of 139) were positive for GAD65 autoantibodies at diagnosis of GDM compared to $0 \%$ (0 of 27) of healthy pregnant women. All 3 GAD65 autoantibody positive GDM patients subsequently developed IDDM after a median of 14 months (range 4-34). GAD65 autoantibodies
\end{abstract}

were present in $50 \%$ (14 of 28 ) of sera from women with IDDM diagnosed during pregnancy. The non-insulin-requiring remission period was significantly shorter in GAD65 autoantibody positive patients (median 0.5 years [range 0-6.0 years]) than in GAD65 antibody negative patients (median 2.6 years; range $0-9.7$ years; $p<0.05$ ). The results suggest that screening for GAD65 autoantibodies in women with GDM or IDDM diagnosed during pregnancy may be useful for predicting the clinical course of the disease. [Diabetologia (1996) 39: 1329-1333]

Keywords GAD65, IAA, ICA, HLA, gestational diabetes, pregnancy.
During pregnancy there is an increased risk of developing glucose intolerance, a phenomenon referred to as gestational diabetes mellitus (GDM). GDM complicates $1-3 \%$ of all pregnancies and is normally managed satisfactorily by dietary treatment alone $[1$, 2]. The occurrence of GDM primarily predisposes to the development of non-insulin-dependent diabetes (NIDDM) [3], but it is also associated with a significant risk of later development of insulin-dependent diabetes mellitus (IDDM) [3-5]. In a group of Danish

Received: 31 January 1996 and in revised form: 8 May 1996

Corresponding author: Dr. J.S. Petersen, Zymogenetics, 1201 Eastlake Avenue East, Seattle WA., 98102, USA

Abbreviations: GDM, Gestational diabetes mellitus; IDDM, insulin-dependent diabetes mellitus; GAD, glutamic acid decarboxylase; ICA, islet cell antibodies; OGTT, oral glucose tolerance test; NIDDM, non-insulin-dependent diabetes mellitus; GABA, gamma-amino-butyric acid
GDM patients [5] 3.7\% (9 of 241) developed IDDM during a median of 6 years' follow-up (range 211 years). In this study the incidence of IDDM in patients with a history of GDM was more than 40 times higher than that found in the background population [4]. Patients with GDM therefore provide an interesting opportunity to assess the predictive value of autoantibodies to islet cell antigens for progression to disease.

During pregnancy there is also an increased risk of developing IDDM [6], which always requires insulin treatment in contrast to most cases of GDM. However, after pregnancy, there is often a longer non-insulin-requiring remission period than in non-pregnant IDDM patients (years compared to months) [7]. This remission period resembles a prediabetic phase, and as for the GDM patients, provides a unique opportunity to assess the predictive value of autoantibodies to islet cell antigens for loss of beta-cell function. 
One of the most thoroughly studied autoimmune phenomena associated with the development of IDDM are the circulating autoantibodies which react with the gamma-amino-butyric acid (GABA) synthesizing enzyme glutamic acid decarboxylase (GAD65) [8-13]. We have recently demonstrated that the presence of GAD65 autoantibodies, but not islet cell antibodies (ICA), was predictive for a more rapid loss of beta-cell function after clinical onset of IDDM during the subsequent 12 months [13].

Thus, in the present study, we have correlated the presence and level of GAD65 antibodies with the progression or recurrence of the disease, using serum samples from well-defined groups of women with either GDM or IDDM diagnosed during pregnancy in order to further characterize the association of GAD65 autoantibodies with the beta-cell destructive process in IDDM.

\section{Subjects and methods}

Patients. We have studied sera from 28 women with IDDM diagnosed during pregnancy between $1.5-16.0$ years after delivery (median 7.7 years). The clinical data of the mothers have previously been reported [14].

At the time of the present study all of the mothers had IDDM diagnosed according to World Health Organization criteria and were thus insulin treated, whereas all the children were free of diabetes. The mothers' HLA-DR3/DR4 tissue type distribution was the same as that of non-pregnant IDDM patients and differed from that of a control group from the background population [15].

Details pertaining to the GDM patient population and the general design of the study have recently been published [5]. Briefly, 355 women with GDM were diagnosed between 1978 and 1985 at the Diabetes Centre, Rigshospitalet, Copenhagen. Diagnosis of GDM was confirmed by a 3-h, 50-g oral glucose tolerance test (OGTT), which was considered abnormal if two or more glucose values exceeded the mean $+3 \mathrm{SD}$ value of a group of 46 normal non-pregnant control subjects without family history of IDDM [16] investigated using the same procedure. Of the GDM women 298 were treated satisfactorily with diet alone and 241 of the women together with 57 healthy control women participated in a follow-up examination 211 years after pregnancy. The women in the control group had a normal OGTT during the last trimester of pregnancy (studied during the years 1978-1985) and were non-obese. Serum samples from an index pregnancy were available for 139 women with GDM and 27 control subjects. At follow-up, serum samples were collected from 133 women with previous GDM and 27 control subjects 4-11 years (median 6.3) and 5-10 years (median 7.6), respectively after the index pregnancy. Women, who at follow-up had been treated with insulin, were investigated with an intravenous glucagon test to evaluate residual beta-cell function while all the other women were investigated by a 75-g OGTT. Serum samples from during pregnancy and follow-up were assayed for GAD65 autoantibodies. The subgroup of previous GDM women investigated for GAD65 autoantibodies were representative for the whole group of GDM patients with respect to baseline variables including age, parity, family history of diabetes, body mass index (BMI), and fasting plasma glucose.
Serum samples obtained from IDDM patients $(n=50)(22$ women) within 2 weeks after diagnosis (mean age 21 years, range 15-60) were obtained from the Steno Diabetes Center. Sera from healthy control individuals $(n=50)$ without diabetes were age matched to sera from the diabetic patients.

GAD65 antibody assay. GAD65 autoantibodies were measured as previously described [17]. Briefly, the human GAD65 cDNA [18] was transcribed and translated in vitro according to the manufacturer's instructions (Promega, Madison, Wis., USA), in the presence of $\left[{ }^{35} \mathrm{~S}\right]$ methionine (Amersham, Amersham, Bucks., UK). The in vitro translated GAD65 was applied to an NAP 5 column (Pharmacia, Uppsala, Sweden) in order to remove free $\left[{ }^{35} \mathrm{~S}\right]$ methionine and aggregates. Aliquots containing approximately $30000 \mathrm{cpm}$ of in vitro translated GAD65 were used for immunoprecipitation. The immune complexes were isolated with Protein A-Sepharose (Pharmacia) and transferred to individual wells in a 96-well filtration system (Multiscreen; Millipore, Bedford, MA, USA). After 10 washings, each filter was counted in a scintillation counter (Packard Tri-carb 4530, Downers Grove, IL, USA), and the amount of immunoprecipitated GAD65 was quantified. Sera were regarded as positive when the GAD65 autoantibody cpm exceeded the mean plus 3 SD of GAD65 autoantibody indices in the healthy control groups.

In the 1995 International Diabetes Workshop (IDW) combined autoantibody workshop the specificity was $98 \%$ and sensitivity was $87 \%$ (<30 years) for this assay at our laboratory.

\section{Statistical analysis}

Statistical analyses included Spearman's correlation analysis, chi square, life-table analysis, Mann-Whitney U-test and Fisher's exact test. Significance was considered at the level of $5 \%$.

\section{Results}

GAD65 autoantibodies in patients with GDM. Among the patients with GDM, $4.3 \%$ (6 of 139) developed IDDM and $10.7 \%$ (15 of 139) developed NIDDM, while none of the control subjects developed diabetes [4]. GAD65 autoantibodies in serum from the healthy control subjects, in the GDM patients at diagnosis, i.e. at pregnancy, and at follow-up were analysed. Of the 139 patients with GDM $2.2 \%$ (3 of 139), but none of the control subjects were found to be GAD65 autoantibody positive during the index pregnancy (Table 1). All 3 GAD65 autoantibody positive GDM patients developed IDDM after a median of 14 months (range 4-34 months). None of the GDM patients without IDDM at follow-up $(n=133)$ were GAD65 autoantibody positive. (GDM patients with IDDM compared to GDM patients without IDDM $p<0.001)$. At the follow-up 4-11 years (median 6.3) after pregnancy, GAD65 autoantibodies were still present in 2 of the 3 women who had been GAD65 autoantibody positive during pregnancy (not shown). None of the control subjects or the initially GAD65 autoantibody negative GDM 
Table 1. ICA [4] and GAD65 autoantibodies in pregnant women with or without GDM

\begin{tabular}{llll}
\hline & $\begin{array}{l}\text { ICA } \\
\text { positive } \\
(n / \text { total })\end{array}$ & $\begin{array}{l}\text { GAD65 } \\
\text { positive } \\
(n / \text { total })\end{array}$ & $\begin{array}{l}\text { ICA and/ } \\
\text { or GAD65 } \\
\text { positive } \\
(n / \text { total })\end{array}$ \\
\hline GDM - IDDM & $1 / 133$ & $0 / 133$ & $1 / 133$ \\
GDM + IDDM & $3 / 6^{\mathrm{a}}$ & $3 / 6^{\mathrm{a}}$ & $4 / 6^{\mathrm{a}}$ \\
Controls & $0 / 27$ & $0 / 27$ & $0 / 27$ \\
\hline
\end{tabular}

GDM + IDDM, Previous GDM patients who developed IDDM at follow-up 4-11 years after pregnancy (median 6.3 years). GDM-IDDM, previous GDM patients with no IDDM at follow-up. Control subjects, healthy pregnant women. None of the GDM patients or control subjects were positive for IAA [4]. Two of the GAD65 autoantibody positive patients were positive for ICA [4]. ${ }^{\mathrm{a}} p<0.001 \mathrm{GDM}$ patients with IDDM vs GDM patients without IDDM

patients were positive for GAD65 autoantibodies at follow-up.

GAD65 antibodies in patients with IDDM diagnosed during pregnancy. GAD65 autoantibodies were detected in $50 \%$ (14 of 28 ) of women with IDDM diagnosed during pregnancy. In comparison, $72 \%$ (36 of 50) recent onset IDDM patients were positive for GAD65 autoantibodies compared to $0 \%$ (0 of 50) of the healthy control subjects (Fig. 1). The 28 women with IDDM diagnosed during pregnancy were treated without insulin therapy after an average of 1.5 years (range $0-9.7$ ) after delivery. The non-insulin-requiring remission period was shorter in patients who were GAD65 autoantibody positive (14 of 28) (median 0.5 years, range 0-6.0) than in GAD65 autoantibody negative patients (14 of 28) (median 2.6 years, range $0-9.7)(p<0.05)$ (Fig. 2$)$. Sera for GAD65 autoantibody analysis were collected 7.1 and 7.8 years after pregnancy for the GAD65 autoantibody positive or negative groups of patients, respectively.

There was no correlation between the level of GAD65 autoantibodies and the duration of the noninsulin-requiring remission period $(p=0.8876)$ (data not shown). No association between the level and prevalence of GAD65 autoantibodies and HLA class I and HLA-DR was observed (data not shown).

\section{Discussion}

The prevalence of GAD65 autoantibodies was only $2.2 \%$ (3 of 139) in women with diet-treated GDM. However, the presence of GAD65 autoantibodies during pregnancy was associated with a very high risk of subsequent development of IDDM. Thus, at follow-up 6 years after the index pregnancy (when IDDM incidence is more than 40 times increased in women who previously had GDM compared to the

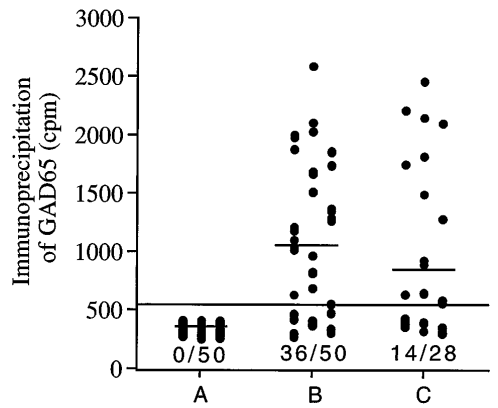

Fig. 1. GAD65 autoantibodies in sera analysed by immunoprecipitation using in vitro translated $\left[{ }^{35} \mathrm{~S}\right]$ methionine labelled human islet GAD65. A Healthy control subjects $(n=50)$; B recent-onset IDDM patients $(n=50)$; C patients who developed IDDM during pregnancy $(n=50)$. The cut-off value is represented by the solid line (mean $+3 \mathrm{SD}$ of the healthy control subjects). Mean cpm in each group is indicated with a solid line. There was no significant difference in the prevalence or level of GAD65 autoantibodies between groups B and C

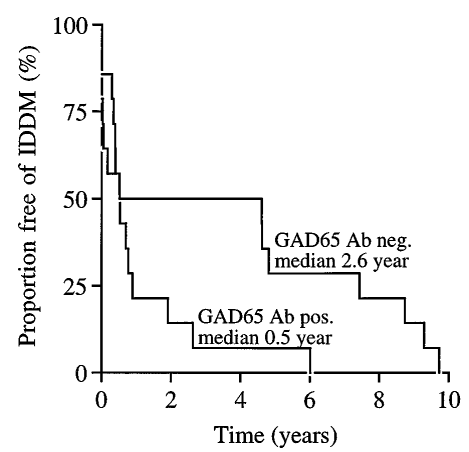

Fig. 2. Predictive value of GAD65 autoantibody status for the prevalence of non-insulin-requiring remission in patients with IDDM diagnosed during pregnancy. Prevalence of non-insulin-requiring remission in patients who were positive for GAD65 autoantibodies $(n=14)$ or negative for GAD65 autoantibodies $(n=14)$. The median non-insulin-requiring remission period in GAD65 autoantibody positive patients was 0.5 years (range 0-6.0 years) compared to 2.6 years (range 0 9.7 years) in GAD65 autoantibody negative patients (life-table analysis showed no significant difference between the two groups, between 1.9-4.61 years after delivery, $p<0.05$ )

general Danish population [4]) all women with previous GDM who were positive for GAD65 autoantibodies had developed IDDM. In contrast, none of the age-matched healthy control subjects, GDM patients without IDDM or NIDDM or GDM patients who had developed NIDDM during follow-up were positive for GAD65 autoantibodies. In an earlier study [4] sera from the same group of GDM patients were analysed for insulin autoantibodies (IAA) and ICA. None were positive for IAA, including the 6 individuals who later developed IDDM. However, the age of onset in the women who developed IDDM after pregnancy was median 24.0 years (range 18-36) and IAA is normally associated with early onset of 
IDDM [13]. In contrast, $2.9 \%$ (4 of 139) of the GDM patients were positive for ICA and during follow-up 3 of these women developed IDDM [4]. Four of the 6 individuals were positive for ICA and/or GAD65 autoantibodies (Table 1). The low prevalence of islet cell autoantibodies, i.e. GAD65 and ICA, found in this patient material is in contrast to previous studies, where a prevalence between $1-18 \%$ has been reported [19-21]. The most likely explanation for this low prevalence of islet cell autoantibodies is that we, in contrast to many previous studies, excluded insulin-treated GDM patients and therefore investigated a more homogeneous group of GDM patients. This explanation is supported by Freinkel et al. [20] who found that the prevalence of ICA increased with an increasing degree of glucose intolerance, and thus an increasing frequency of insulin-treatment and hence an increasing risk of IDDM. Furthermore, it has recently been demonstrated, by comparing the prevalence of GAD65 autoantibodies in insulin-treated GDM patients with non-insulin-treated GDM patients that the prevalence of GAD65 autoantibodies is $15 \%$ (5 of 32) and $1 \%$ ( 1 of 80$)$, respectively [22].

Previously, we have demonstrated that beta-cell function deteriorated more rapidly after clinical onset of IDDM in patients who were GAD65 autoantibody positive at onset than in those who were GAD65 autoantibody negative [13]. IDDM diagnosed during pregnancy is often characterized by a long remission period after delivery, which is probably due to the high insulin demand in pregnancy [6]. Therefore, such patients provide a unique opportunity to study the correlation between GAD65 autoimmunity and the final stages of beta-cell destruction. In the present study, we found that there was a difference in the non-insulin-requiring remission period in patients who were GAD65 autoantibody positive (median 0.5 years) compared to patients who were GAD65 antibody negative (2.6 years), respectively (Fig. 2), thus supporting our previous findings [13]. The sera from the patients with IDDM diagnosed during pregnancy were obtained several years after delivery; however, $50 \%$ (14 of 28 ) were still positive for GAD65 autoantibodies, indicating that GAD65 autoantibodies are very persistent. In agreement with these data, we have recently demonstrated that $57 \%$ (38 of 67 ) of IDDM patients with a mean duration of IDDM of more than 25 years are still positive for GAD65 autoantibodies compared to only $21 \%$ (14 of 67) who are ICA positive [23]. The small decrease in GAD65 autoantibodies with time could potentially change the outcome of this study compared to a study where the sera were collected at onset of disease. However, we have previously demonstrated, by analysing 139 resent-onset IDDM patients, that only the presence and not the level of GAD65 autoantibodies is correlated to a rapid decrease in beta-cell function after onset. Likewise, in the present study there was no significant correlation between the level of GAD65 autoantibodies and the length of the non-insulin-requiring remission (data not shown). Thus, it could be speculated that the non-insulin-requiring remission period observed in the GAD65 autoantibody negative group is probably underestimated, since this group contained patients who had initially been GAD65 autoantibody positive.

In conclusion, the lack of autoantibodies in the majority of women with GDM supports the notion that GDM is not caused by an autoimmune process as is the case with IDDM. However, women with GDM who have islet cell autoantibodies, i.e. GAD65 and/or ICA, have a particularly high risk of developing IDDM later in life. Furthermore, we have demonstrated that the presence of GAD65 autoantibodies in patients with IDDM diagnosed during pregnancy is predictive for a more rapid loss of beta-cell function. Taken together, these results suggest that it should be considered whether pregnant women who develop GDM or IDDM should be analysed for the presence of GAD65 autoantibodies since this may help to identify those individuals who might benefit from preventive treatments.

Acknowledgements. We are indebted to MS E. J. Jensen, MS C. Kühl, MS M. L. Borgen, MS L. Poulsen, and MS U. Jensen for technical assistance. J.S. P. is supported by the Juvenile Diabetes Foundation and P.D. by the Novo Foundation and the Danish Diabetes Association.

\section{References}

1. The Third International Workshop Conference on Gestational Diabetes Mellitus (1991) Summary and recommendations. Diabetes 40: 197-201

2. Gabbe S (1986) Gestational diabetes mellitus. N Eng J Med 315: $1025-1026$

3. O'Sullivan JB (1989) The Boston gestational diabetes studies: review and perspectives. In: Sutherland HW, Stowers JM, Pearson DWM (eds) Carbohydrate metabolism in pregnancy and the newborn. Springer-Verlag, Berlin pp 287-294

4. Damm P, Kühl C, Buschard K et al. (1994) Prevalence and predictive value of islet cell antibodies and insulin autoantibodies in women with gestational diabetes. Diabet Med 11: 558-563

5. Damm P, Kühl C, Bertelsen A, Mølsted-Pedersen L (1992) Predictive factors for development of diabetes in women with previous gestational diabetes. Am J Obstet Gynecol 167: 607-616

6. Buschard K, Buch I, Mølsted-Pedersen L, Hougaard P, Kühl C (1987) Increased incidence of true type 1 diabetes acquired during pregnancy. BMJ 294: 275-279

7. Buschard K, Hougaard P, Mølsted-Pedersen L, Kühl C (1990) Type 1 (insulin-dependent) diabetes mellitus diagnosed during pregnancy: a clinical and prognostic study. Diabetologia 33: 31-35

8. Bækkeskov S, Aanstoot H-J, Christgau S et al. (1990) Identification of the $64 \mathrm{~K}$ autoantigen in insulin dependent 
diabetes as the GABA-synthesising enzyme glutamic acid decarboxylase. Nature 347: 151-156

9. Hagopian WA, Karlsen AE, Gottsater A et al. (1993) Quantitative assay using recombinant human islet glutamic acid decarboxylase (GAD65) shows that $64 \mathrm{~K}$ autoantibody positivity at onset predicts diabetes type. J Clin Invest 91: 368-374

10. Thivolet CH, Tappaz M, Durand A et al. (1992) Glutamic acid decarboxylase (GAD) autoantibodies are additional predictive markers of type 1 (insulin-dependent) diabetes mellitus in high risk individuals. Diabetologia 35: 570-576

11. De Aizpurua HJ, Wilson YM, Harrison LC (1992) Glutamic acid decarboxylase autoantibodies in preclinical insulin-dependent diabetes. Proc Natl Acad Sci USA 89: 98419845

12. Bingley PJ, Christie MR, Bonifacio E et al. (1994) Combined analysis of autoantibodies improves prediction of IDDM in islet cell antibody-positive relatives. Diabetes 43: $1304-1310$

13. Petersen JS, Dyrberg T, Karlsen AE, Mølvig J, Nerup J, Mandrup-Poulsen T and the Canadian-European Randomized Control Trial Group (1994) Glutamic acid decarboxylase (GAD65) autoantibodies in prediction of beta-cell function and remission in recent onset insulin-dependent diabetes mellitus following cyclosporin-treatment. Diabetes 43: 1291-1296

14. Buschard K, Mølsted-Pedersen L, Palmer J, Kühl C, Lund E, Bottazzo GF (1989) Investigation in children who were in utero at onset of insulin-dependent diabetes in their mothers. Lancet (8642): 811-814

15. Møller-Jensen B, Buschard K, Bush I et al. (1987) HLA association in insulin-dependent diabetes mellitus diagnosed during pregnancy. Acta Endocrinol 116: 387-389

16. Kühl C (1975) Glucose metabolism during and after pregnancy in normal and gestational diabetic women. I.
Influence of normal pregnancy on serum glucose and insulin concentration during basal fasting conditions and after a challenge with glucose. Acta Endocrinol 79: 709-719

17. Petersen J, Hejnæs KR, Moody A et al. (1994) Detection of $\mathrm{GAD}_{65}$ antibodies in diabetes and other autoimmune diseases using a simple radioligand assay. Diabetes 43: 459467

18. Karlsen AE, Hagopian WA, Petersen JS et al. (1992) Recombinant glutamic acid decarboxylase (representing the single isoform expressed in human islets) detects IDDMassociated 64,000-Mr autoantibodies. Diabetes 41: 13551359

19. Catalano PM, Tyzbir ED, Sims EAH (1990) Incidence and significance of islet cell antibodies in women with previous gestational diabetes. Diabetes Care 13: 478-482

20. Freinkel N, Metzger BE, Phelps RL, Dooley S, Ogata ES, Radvany RM (1985) Gestational diabetes mellitus: heterogeneity of maternal age, weight, insulin secretion, HLA antigens and islet cell antibodies and the impact of maternal metabolism on pancreatic B-cell and somatic development in the offspring. Diabetes 34[Suppl 2]:1-7

21. Fallucca F, Di Mario U, Gargiulo P et al. (1985) Humoral immunity in diabetic pregnancy: interrelationship with maternal/neonatal complications and maternal metabolic control. Diabete Metab 11: 387-395

22. Tuomilehto J, Zimmet P, MacKay IR et al. (1994) Antibodies to glutamic acid decarboxylase as predictors of insulindependent diabetes mellitus before clinical onset of disease. Lancet 343: 1383-1385

23. Zanone MM, Petersen JS, Peakman M et al. (1994) High prevalence of autoantibodies to glutamic acid decarboxylase in long-standing insulin-dependent diabetes is not a marker of symptomatic autonomic neuropathy. Diabetes 43: $1146-1151$ 